\title{
Site-dependent acellularisation effects explain altered tissue mechanics: ultrastructural insights
}

\author{
N. Hammer \\ Department of Anatomy, University of Otago, Lindo Ferguson Building, Dunedin, New Zealand
}

[Received: 22 November 2016; Accepted: 16 December 2016]

\begin{abstract}
Acellular scaffolds are used for the surgical repair of soft tissue injury forming a biological basis for cell remodelling. Previously tissue-dependent effects sodium-dodecyl-sulphate (SDS) have been determined on the extracellular matrix (ECM) of different tissue types. This short report aims at extending these findings onto a tissue-layer level with focus on the ECM. Porcine ureters, oesophagiand skin undenwent acellularisation using SDS, whereas control samples remained in a native condition. The samples were investigated histologically and ultrastructurally electron microscopy. Dense collagen bundles were seen in all native samples throughout the layers, and moderate to strong decreases in collagen density in the acellular state, accompanied by clumping. Collagen bundles were altered differently. Transition from straightened into coiled alignment was observed in the ureters' intima and all oesophageal layers, the opposite was observed in the ureters' media and adventitia. Skin samples appeared discontinuously following acellularisation, with collagens curling in the subcutis and dermis and disruptions in the subepidermis. Collagen fibre integrity appeared unchanged. SDS-related alterations include tissue- and site-dependent alterations of the collagen bundles. These insights into the ECM provide further explanation of acellularisation-induced change in mechanical properties, resulting in increased stiffness in ureters, and stiffness in skin. (Folia Morphol 2017; 76, 3: 355-360)
\end{abstract}

Key words: decellularisation, mechanical testing of soft tissues, scanning electron microscopy, tissue layer, transmission electron microscopy

\section{INTRODUCTION}

Acellular tissues are becoming increasingly used as a source for surgical soft tissue reconstruction $[1,2,7]$. Being freed of cells and cellular components, these scaffolds produce minute antigenicity $[2,6,9]$ and provide a biologic environment for cellular ingrowth and subsequent remodelling. However, there is a lack of morphologic and functional data on how exactly the commonly used methods of acellularisation impact extracellular matrix integrity, including commonly used chemical and physical protocols $[3,10]$.
In a previous study, we compared ureter, oesophagus and skin specimens in a native condition and after acellularisation using sodium-dodecyl-sulphate (SDS) [8]. We found different effects of acellularisation onto the mechanical properties of these tissues. Ureters and oesophagi showed increases of elastic modulus and ultimate stress, whereas skin samples showed decreases in these values. These tissue-dependent characteristics in mechanical strength could partially be explained by altered cross-section and interruption of collagen continuity by removed hair follicles. However, ambiguity

Address for correspondence: N. Hammer, MD, Department of Anatomy, University of Otago, Lindo Ferguson Building, 270 Great King St Dunedin 9016, New Zealand, tel: +64 3479 7362, fax: +64 3479 7254, e-mail: nlshammer@googlemail.com 


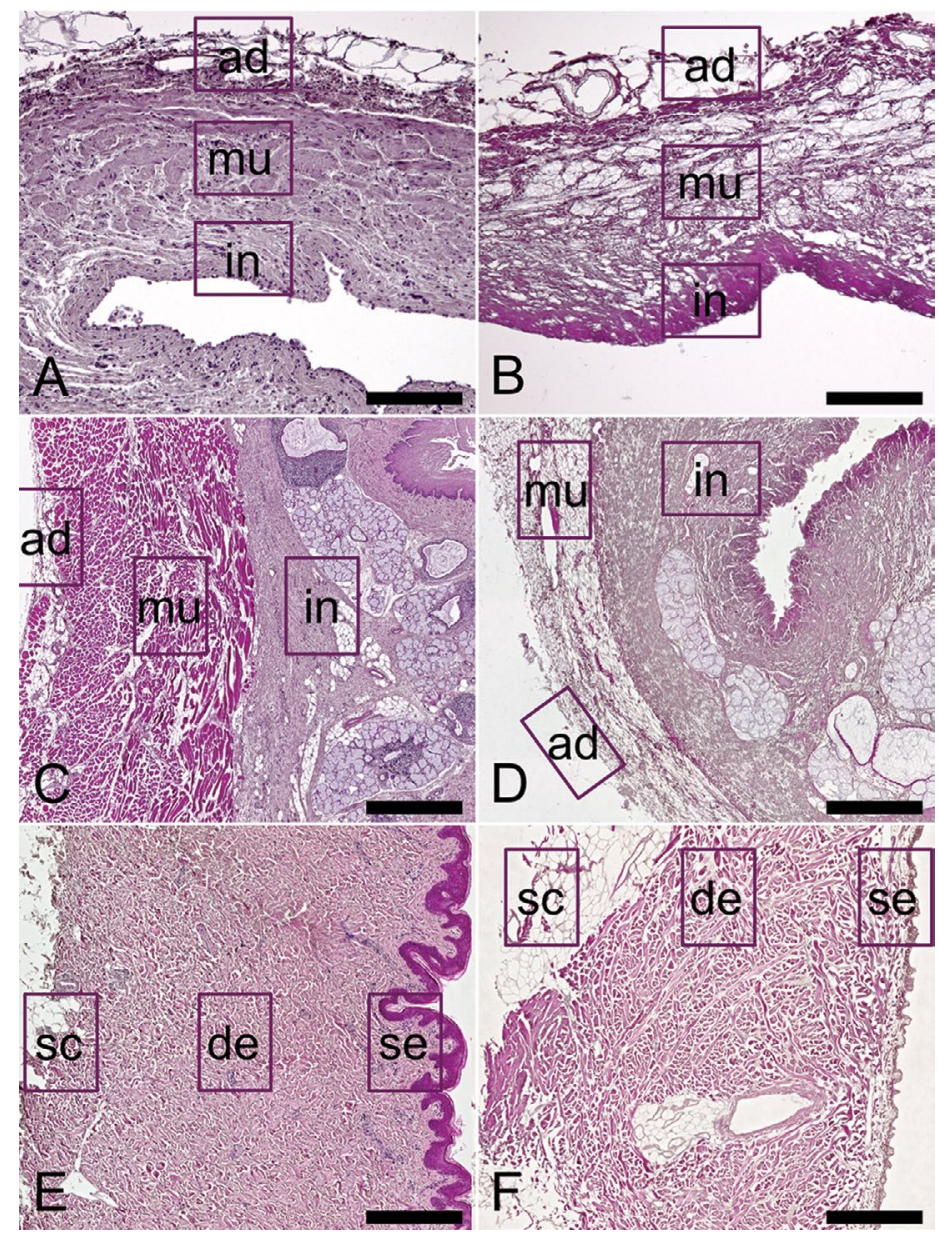

Figure 1. Native (left) and acellular tissues (right) in haematoxylin-eosin staining, $10 \times$ magnification. Ureter (A, B), oesophagus (C, D) and skin (E, F); ad — tunica adventitia; de — dermis; in — tunica intima; mu tunica muscularis; sc - subcutis; se - subepidermis; scale bar $100 \mu \mathrm{m}$. persists regarding the morphological alterations throughout all tissue layers of the respective organs and regiondependent homogeneity, which was the rationale for this follow-up study. The given preliminary report aimed at substantiating these previous findings in porcine ureters, oesophagi and skin. For this purpose, the tissues were investigated ultrastructurally considering the relevant tissue layers to further elucidate site-dependent effects of SDS-based acellularisation, with particular focus onto the formed parts of the extracellular matrix (ECM).

\section{MATERIALS AND METHODS}

The tissues were obtained from 4- and 7-monthold pigs post mortem approved by the local animal welfare committee of Leipzig, Germany (TVV 38/12). Specimens were obtained from the oesophagus, ureter and skin as part of a larger study [8].

\section{Acellularisation and histology}

The acellularisation was carried out according to Schleifenbaum et al. [8]. The specimens were sub- merged in SDS for 7 days in case of the oesophagi and ureters and for 28 days in case of the skin specimens. Following this, the samples were rinsed 7 days in distilled water and then shock frozen. Samples of these tissues were fixed in paraformaldehyde, paraffin-embedded and sectioned at $5 \mu \mathrm{m}$ (Fig. 1). Haematoxylin-eosin (HE) staining was carried out as reported previously [4].

\section{Scanning and transmission electron microscopy}

Samples measuring $5 \mathrm{~mm}^{3}$ were prepared from the tunica intima, media and adventitia of the ureters and oesophagi of both the native and acellular tissues, as indicated in Figure 1. Subepidermal, dermal and subcutis samples were removed from the skin (Fig. 1). The tissues were fixed in 3\% glutaraldehyde/ $0.1 \mathrm{M}$ phosphate buffer ( $\mathrm{pH} 7.4$ ) and washed in distilled water. The samples for scanning electron microscopy (SEM) were dehydrated, followed by the critical point drying process (CPD-030, Bal-Tec AG, Liechtenstein). SEM images were taken with a JSM-6700F field emission microscope (JEOL Ltd., Tokyo, Japan) at magnifications between 


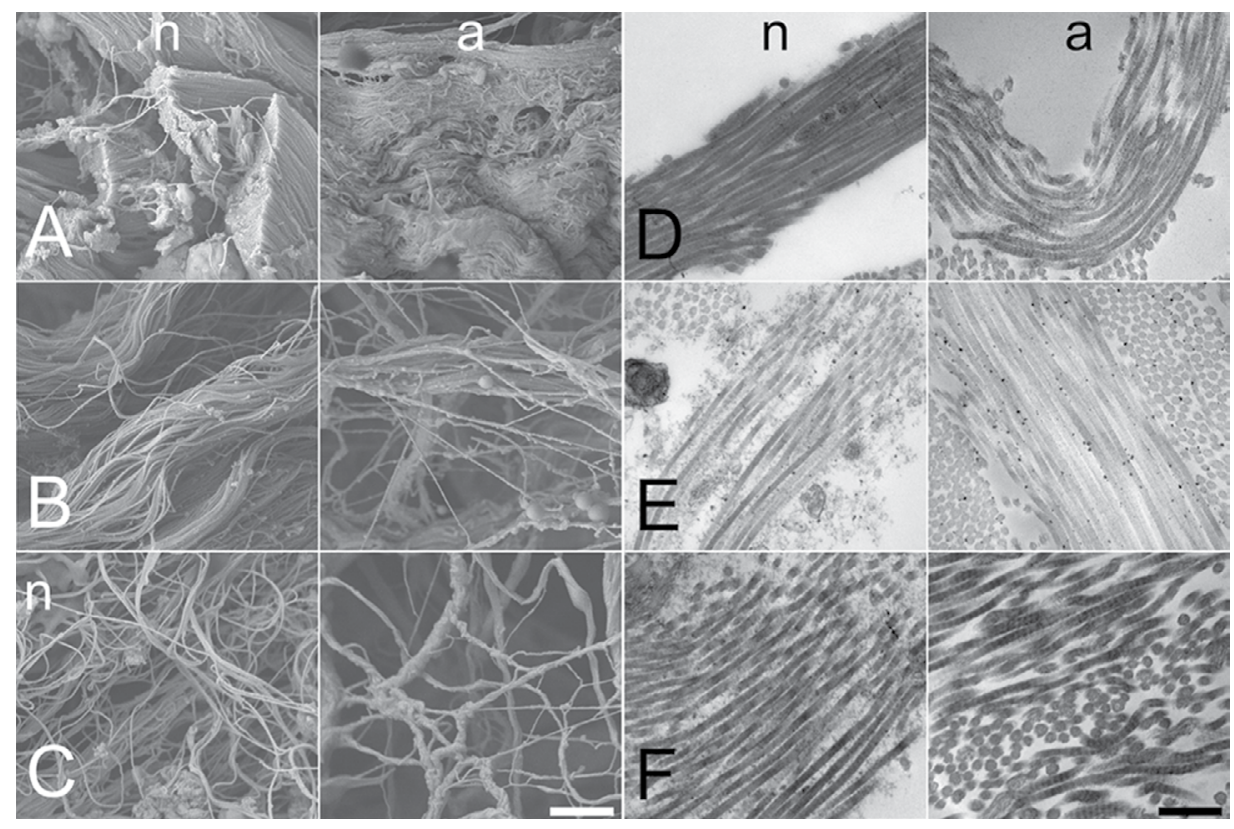

Figure 2. Native (n) and acellular ureter (a) under scanning electron microscopy (A-C; scale bar $2 \mu \mathrm{m}$ ) and transmission electron microscopy (D-F; scale bar $500 \mathrm{~nm}$ ). Intima (A, D), media (B, E), adventitia (C, F).
$50 \times$ and $50,000 \times$. The samples for transmission electron microscopy (TEM) were washed in $0.1 \mathrm{M}$ cacodylate buffer, post-fixed in 1\% osmium tetroxide, washed in distilled water, and dehydrated and infiltrated in ascending ethanol series, propylene oxide and resin. Semi-thin sections of $1 \mu \mathrm{m}$ were used for alignment before cutting ultrathin sections and contrasted with uranyl acetate and lead citrate. A Philips CM100 BioTWIN (Philips/FEI Corporation, Eindhoven, Netherlands) equipped with a MegaView III digital camera (Olympus Soft Imaging Solutions $\mathrm{GmbH}$, Münster, Germany) was used to obtain TEM images at 6600-66,000× magnifications.

\section{RESULTS}

The boxes in Figure 1 indicate the anatomical regions from which the samples for electron microscopy were obtained. Acellularisation caused an extensive removal of the cells and cellular components in the ureter, oesophagus and skin.

\section{Electron microscopy revealed not only tissue-, but also site-specific alterations of type collagen bundles caused by SDS}

In the tunica intima of the native ureters, a solid network of straightly-aligned collagen bundles was observed (Fig. 2A; Table 1). In the acellular condition, a marked thinning of the collagen density was observed (Fig. 2A), accompanied by a coiled appearance of the collagen bundles. Regarding the media (Fig. 2B) and the adventitia (Fig. 2C) of the ureters, a quantitative loss of collagens bundles was seen together with a marked clotting. The loss in bundle density was more marked in the media than in the adventitia. In both the media and the adventitia, the collagen bundles were coiled in the native and straightened in the acellular condition, which in combination with the now apparent elastins left behind a spider web-like network. Regarding the fibrillar level, no change was observed related to the acellularisation in TEM with the 67-nm bands without marked fibril breakage (Fig. 2D-F; Table 1).

The intima (Fig. 3A; Table 1) and media (Fig. 3B) of the oesophagus specimens both showed a moderate loss of collagen fibres, accompanied by extensive coiling in the acellular state, and clumping in the media. The adventitia (Fig. $3 \mathrm{C}$ ) revealed extensive clumping and loss of collagen bundle structural integrity to some extent, leaving behind elastic fibres. Higher magnifications in TEM showed intact collagen fibres with the typical 67-nm bands (Fig. 3D-F; Table 1). No marked collagen fibril breakage was observed.

In the skin samples a general trend towards collagen fibre clumping was observed, being more pronounced in the subcutaneous (Fig. 4A) than in the dermal (Fig. 4B) and subepidermal regions (Fig. 4C; Table 1). The native collagen bundles in the subdermal and dermal regions had a spaghetti-like appearance, turning into a spiral-like appearance following acellularisation. Beyond emptied hair follicles and adjacent root sheaths in the subepidermal region, a distinct loss of the keratin-rich layer was seen from the native to the acellular condi- 
Folia Morphol., 2017, Vol. 76, No. 3

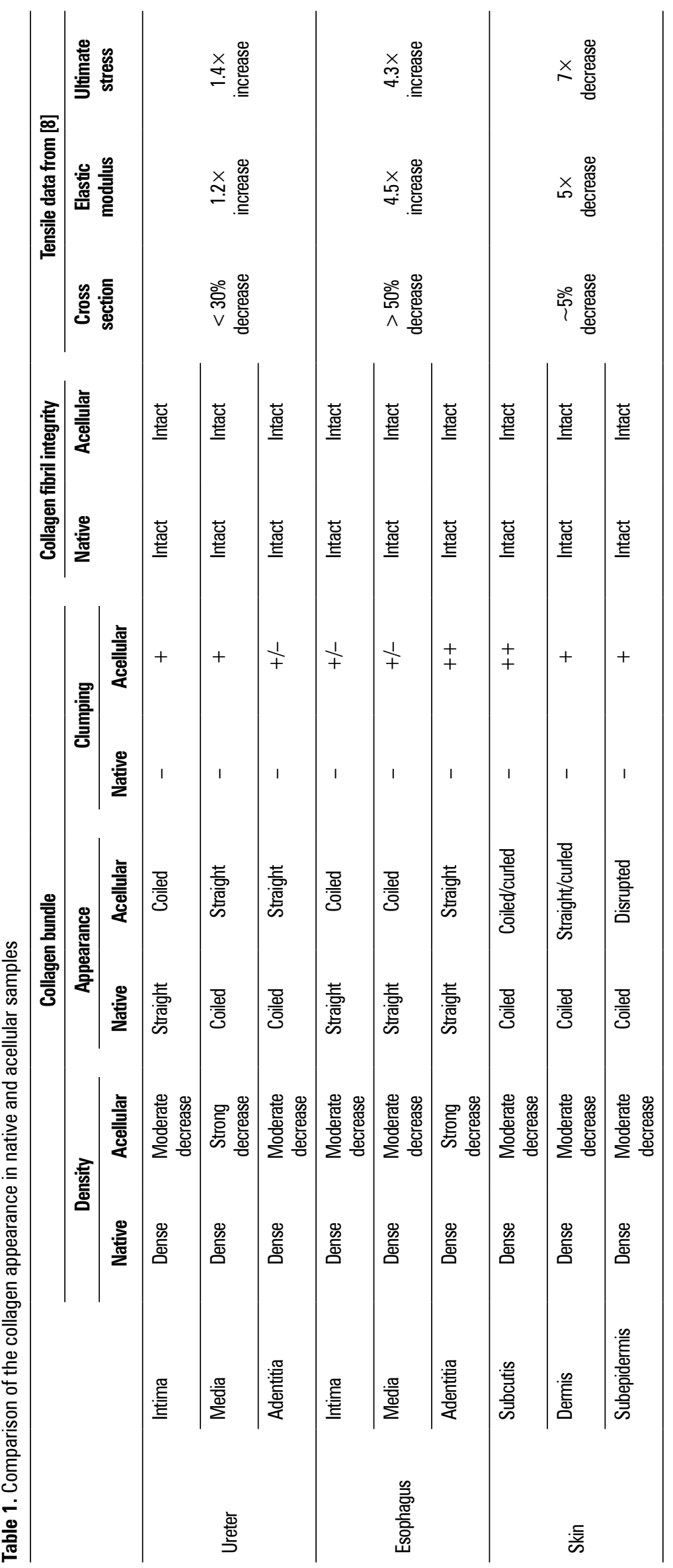




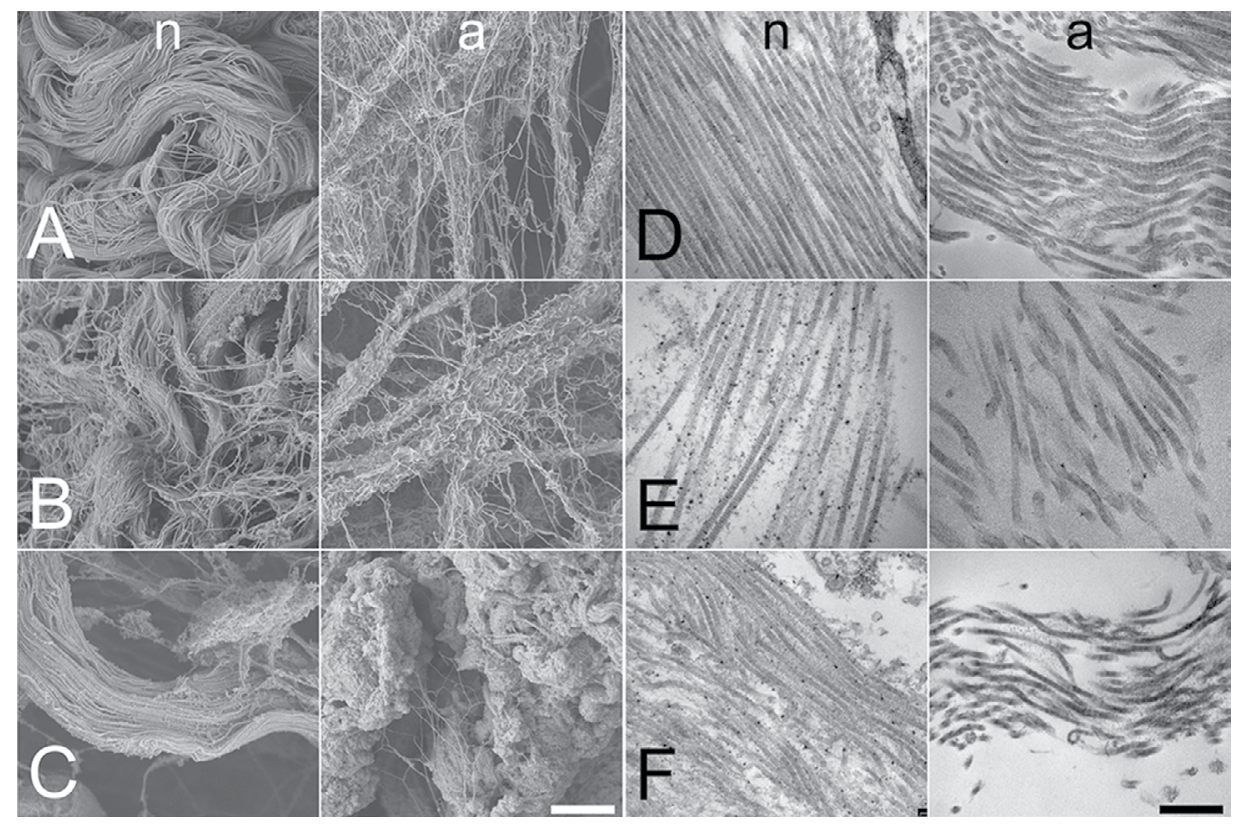

Figure 3. Native (n) and acellular oesophagus (a) under scanning electron microscopy (A-C; scale bar $2 \mu \mathrm{m})$ and transmission electron microscopy (D-F; scale bar $500 \mathrm{~nm})$. Intima $(\mathbf{A}, \mathbf{D})$, media $(\mathbf{B}, \mathbf{E})$, adventitia (C, F).

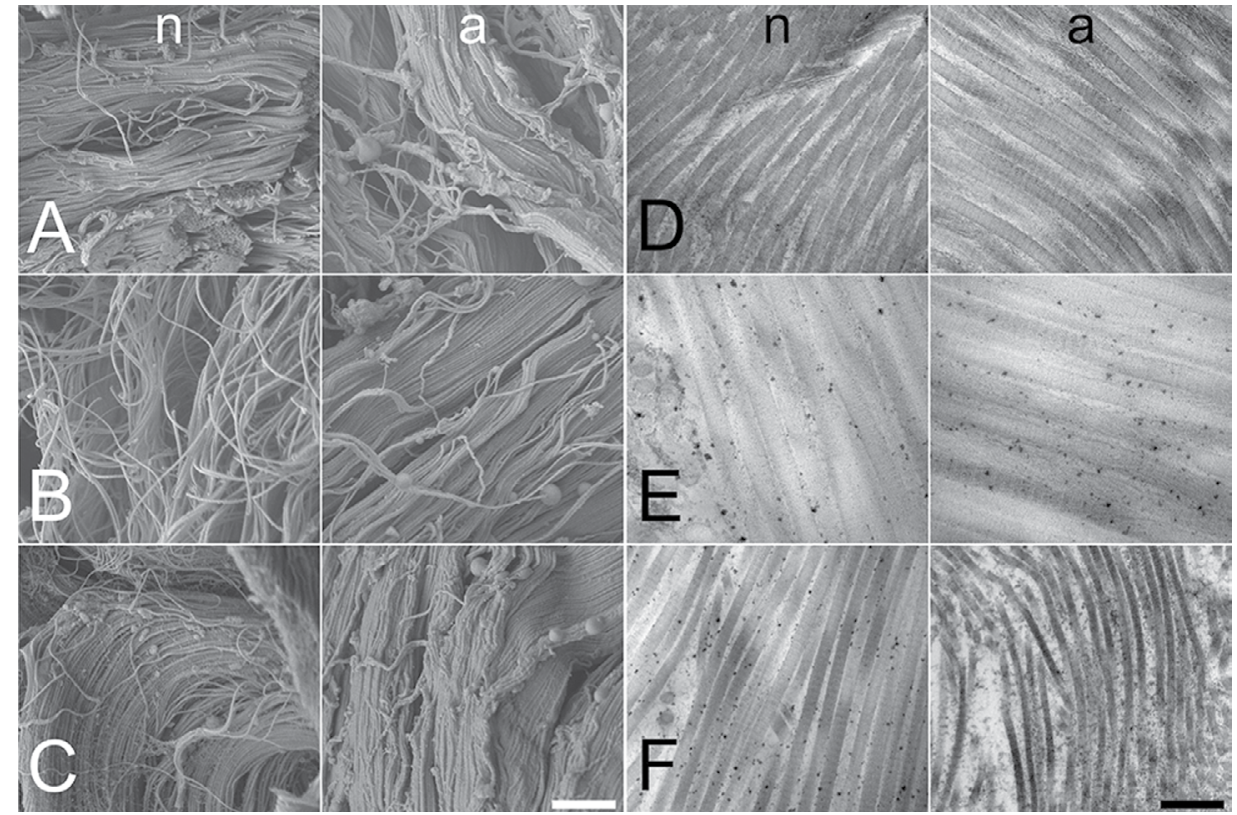

Figure 4. Native (n) and acellular skin (a) under scanning electron microscopy (A-C; scale bar $2 \mu \mathrm{m}$ ) and transmission electron microscopy (D-F; scale bar $500 \mathrm{~nm})$. Subcutis (A, D), dermis (B, E), subcutis (C, F). tion (Fig. 4C). TEM on the fibrillar level again showed the typical 67-nm bands and no signs of breakage, but markedly different fibre diameters depending on the tissue layer (Fig. 4D-F; Table 1).

\section{DISCUSSION AND CONCLUSIONS}

The given study aimed at giving an ultrastructural insight into the effects of SDS-based acellularisation onto porcine ureters, oesophagi and skin. A particular focus was laid onto then collagens being a predominant part of the formed extracellular matrix of these tissues and the backbone of tensile stability. To date there are very few studies on the effects of acellularisation on the micro- and nano-structural integrity of scaffolds following acellularisation protocols $[4,5,8]$. The ECM might be regarded as a main mechanical actor of these organs [8]. It could be shown that the extracellular matrix of these tissues was affected site-dependently with distinctive features throughout the tissue layers.

While the three tissues had a dense overall appearance of the ECM in all layers in the native state, 
there was a moderate to strong decrease of collagen density in all the acellular ureter and oesophagus layers as well as the subepidermis correspondingly, and less distinct change in the subcutaneous and dermal layers. Similarly, inconsistent findings were observed for the collagen bundle appearance, with tissue- and layer-dependent variations. Comparison of the tensile data to the collagen bundle appearance throughout the layers indicates that for the ureters, the increase in elastic modulus and ultimate stress [8] can be correlated with the decrease of the cross-sectional area and the moderate to strong decrease in collagen density per volume in the tunica media and adventitia shown here. This change is accompanied by straightened, i.e. pretensioned collagen bundles, whereas the changes in the intima appear to play a minor role. For the oesophagus, morphological alterations in the tunica intima and media appear to govern the significant increase in tensile strength and elasticity, caused by significant decrease in cross section, and a trend towards a more coiled collagen appearance. In these two tissues, collagen bundle density and appearance does not appear to be an indicator of mechanical strength. In the skin samples, however, the general trend towards decreased collagen bundle density, accompanied with a curled or disrupted appearance, may be an indicator of decreased tensile strength. These effects, combined with the stratum corneum removal, and the weakening induced by the emptied hair follicles in the sense of notching effects, might be indicators of the decreased tensile strength [8]. These findings have a strong clinical implication. Acellular matrices have evolved becoming suitable scaffolds for soft tissue reconstruction $[1,2,5-7]$ due to their reduced antigenicity caused by the partial or complete absence of cellular proteins [9]. We could show previously that acellularisation-related effects are tissuedependent [8]. These findings can now be extended by site-dependent effects within the tissues as well. These findings impact the suitability of these tissues for clinical use, taking into account which part of the scaffold or scaffold composite is of highest interest in surgical reconstruction. A number of limitations apply for the given study. First, only a limited number of tissues were examined to the effect that this preliminary study can only make qualitative conclusions. Second, only one protocol for acellularisation was assessed and the type of tissues under investigation was largely limited with particular focus on the formed parts of the ECM, whereas proteoglycans were not addressed in the current setup.

\section{Acknowledgements}

The author would like to thank Gabriela Aust, Andreas Boldt, Judith Craatz and Stefan Schleifenbaum for their help with the tissues. Elizabeth Girvan, Sharon Lequeux and Allan Mitchell assisted with scanning and transmission electron microscopy.

\section{REFERENCES}

1. Barber FA, Aziz-Jacobo J. Biomechanical testing of commercially available soft-tissue augmentation materials. Arthroscopy. 2009; 25(11): 1233-1239, doi: 10.1016/j. arthro.2009.05.012, indexed in Pubmed: 19896044.

2. Chen J, Xu J, Wang A, et al. Scaffolds for tendon and ligament repair: review of the efficacy of commercial products. Expert Rev Med Devices. 2009; 6(1): 61-73, doi: 10.1586/17434440.6.1.61, indexed in Pubmed: 19105781.

3. Guo L, Qu J, Zheng C, et al. Preparation and characterization of a novel decellularized fibrocartilage "book" scaffold for use in tissue engineering. PLoS One. 2015; 10(12): e0144240, doi: 10.1371/journal.pone.0144240, indexed in Pubmed: 26636672.

4. Hammer N, Huster D, Boldt A, et al. A preliminary technical study on sodium dodecyl sulfate-induced changes of the nano-structural and macro-mechanical properties in human iliotibial tract specimens. J Mech Behav Biomed Mater. 2016; 61: 164-173, doi: 10.1016/j.jmbbm.2016.01.018, indexed in Pubmed: 26866452.

5. Koch H, Graneist C, Emmrich F, et al. Xenogenic esophagus scaffolds fixed with several agents: comparative in vivo study of rejection and inflammation. J Biomed Biotechnol. 2012; 2012: 948320, doi: 10.1155/2012/948320, indexed in Pubmed: 22505820.

6. Rao BM, Kamal TT, Vafaye J, et al. Surgical repair of hip abductors. A new technique using Graft Jacket allograft acellular human dermal matrix. Int Orthop. 2012; 36(10): 2049-2053, doi: 10.1007/s00264-012-1630-6, indexed in Pubmed: 22872412.

7. Roehm KD, Hornberger J, Madihally SV. In vitro characterization of acelluar porcine adipose tissue matrix for use as a tissue regenerative scaffold. J Biomed Mater Res A. 2016; 104(12): 3127-3136, doi: 10.1002/jbm.a.35844, indexed in Pubmed: 27465789.

8. Schleifenbaum S, Prietzel T, Aust G, et al. Acellularization-Induced Changes in Tensile Properties Are Organ Specific - An In-Vitro Mechanical and Structural Analysis of Porcine Soft Tissues. PLoS One. 2016; 11(3): e0151223, doi: 10.1371/ journal.pone.0151223, indexed in Pubmed: 26960134.

9. Schulze-Tanzil G, Al-Sadi O, Ertel W, et al. Decellularized tendon extracellular matrix-a valuable approach for tendon reconstruction? Cells. 2012; 1(4): 1010-1028, doi: 10.3390/cells1041010, indexed in Pubmed: 24710540.

10. Tischer T, Aryee $S$, Wexel $G$, et al. Tissue engineering of the anterior cruciate ligament-sodium dodecyl sulfate-acellularized and revitalized tendons are inferior to native tendons. Tissue Eng Part A. 2010; 16(3): 1031-1040, doi: 10.1089/ ten.TEA.2009.0043, indexed in Pubmed: 19845462. 